\title{
Transfer Fabrication of Liquid Crystal Devices with Microgroove and Wall Structure on Plastic Substrate for Flexible In-plane Switching Liquid Crystal Displays
}

\author{
Daisuke Minami ${ }^{\dagger}$ (member), Yosei Shibata ${ }^{\dagger}$, Takahiro Ishinabe $^{\dagger}$ (member), \\ Hideo Fujikake ${ }^{\dagger}$ (member)
}

\begin{abstract}
In this paper, we propose the in-plane switching liquid crystal device with a microgroove and wall structure, fabricated by a transfer method for printable manufacturing of large-area flexible liquid crystal displays. We confirmed that the liquid crystal devices have a higher transmittance than conventional devices, and that a reduction of the thickness of the residual polymer film is necessary to decrease the driving voltage. Similar to the technique used on glass substrates, fabrication of flexible liquid crystal devices using a plastic substrate is possible using silicone elastomer.
\end{abstract}

Keywords: Flexible liquid crystal display, transfer fabrication, in-plane switching, roll-to-roll.

\section{Introduction}

Recently, there has been significant focus on the development of flexible displays using thin, rollable plastic substrates ${ }^{1-3)}$. These characteristics can dramatically improve the portability of large-area displays, for easier carriage into small spaces such as houses compared to conventional flat-panel large-area displays. However, there are still a number of limitations on the realization of flexible large-area displays. First, low-cost manufacturing is required for these to become commonly used devices. The printing technology known as the roll-to-roll process is expected to fulfill this requirement ${ }^{4-5)}$. Furthermore, the devices are required to have high reliability. Most current longlife electronic displays are of a liquid crystal (LC) type, since LC materials do not degrade chemically. There is some potential for established manufacturing technologies for liquid crystal displays (LCDs) to be applied to flexible displays, to provide a low-cost manufacturing process for high reliability devices.

The manufacturing methods available for flexible LCDs include high-temperature thermal treatments based on the formation of thin-film transistors (TFTs), LC alignment layer and photo-resist spacers. However,

Received March 30, 2018; Revised August 30, 2018; Accepted September 13, 2018

†Tohoku University

(Sendai Japan) thermal processes over the glass transition temperature $\left(T_{g}\right)$ will damage the dimensional stability of a plastic substrate. We have previously reported the fabrication of visible-range transparent and thermally stable polyimide substrate, based on the coat-debond method, to overcome this problem ${ }^{6,7)}$. The drawback of this polyimide substrate is not suitable for low-cost and large-area LCDs because it is more expensive than other plastic materials. Ideally, an inexpensive plastic material, such as polyethylene terephthalate (PET) or triacetylcellulose (TAC), is required for low-cost and large-area flexible LCDs. The $T_{g}$ values of these plastic materials are much smaller than that of polyimide, at around $100{ }^{\circ} \mathrm{C}$. Therefore, fabrication without use of a high-temperature thermal process is required, presenting a critical challenge.

It has been reported that organic TFTs can be fabricated under conditions close to room temperature ${ }^{8)}$. The remaining problems are formation of LC alignment layer and spacer structure at low temperature less than $T_{g}$ of inexpensive plastic materials. As the solution of this problems, the fabrication of flexible LC device having microgroove and wall structure has already reported for twist nematic (TN) mode ${ }^{9}$. However, the TN mode is not suitable for sharing visual information with large audiences, due to the narrow viewing angle; therefore, in-plane switching (IPS) mode LC devices are required. The IPS mode has a wide viewing angle because LC molecules are only driven within the 
azimuthal direction. Based on the requirements discussed, the novel manufacturing method for realization of flexible and printable IPS mode LCDs at low temperature is required.

\section{Proposal of structural transfer method}

Here, we propose the novel manufacturing method using transferred formation of microgroove and wall structure for flexible IPS-mode LC devices, as shown in Fig. 1. An ultraviolet (UV) curable monomer is coated on the plastic substrate; then, a mold with microgroove and wall structure is pressed to the plastic substrate and irradiated with UV light. The microgroove and wall structure is aligned the LC molecules ${ }^{10)-11)}$ and sustain the thickness of the LC layer. Hence, formation of the alignment layer and spacer using a thermal process is unnecessary. The proposed technique is effective for fabrication of low-cost and large-area flexible LCDs, because the structural transfer process can be diverted to roll-to-roll manufacturing.

In this paper, we evaluated the electrooptical characteristics of IPS-mode LC devices with microgroove and wall structure fabricated using the structural transfer method. To clarify the adaptability of the proposed method to flexible LCDs, we investigated the LC alignment behavior on the substrate.

\section{Formation and Evaluation of Microgroove and Wall Structure}

To establish the proposed method, we transferred microgroove and wall structure onto a glass substrate. The fabrication procedure for the microgroove and wall structure is shown in Fig. 2. First, we coated a UV curable monomer material (NOA 63; Norland Ltd.) diluted by toluene (mass ratio 1:1) onto a glass substrate with combshaped transparent electrodes (electrode width: $10 \mu \mathrm{m}$, distance between electrodes: $10 \mu \mathrm{m}$ ), using a spin-coating method for 150 seconds at 5, $000 \mathrm{rpm}$. Next, a silicone elastomer mold (polydimethylsiloxane (PDMS); DOW CORNING Corp.), with microgroove and wall structure was placed on the coated monomer; then, UV light with an intensity of $100 \mathrm{~mW} / \mathrm{cm}^{2}$ was irradiated onto the monomer for $3 \mathrm{~min}$, while the mold was pressed (see Fig.2 (b)). To confirm the formation of the transferred structure, confocal microscope images of the silicone elastomer mold surface and polymer (photo-polymerized monomer) film surface were taken, as shown in Fig. 3. The formation of a uniaxially aligned microgroove and cross-shaped wall structure on the polymer surface was

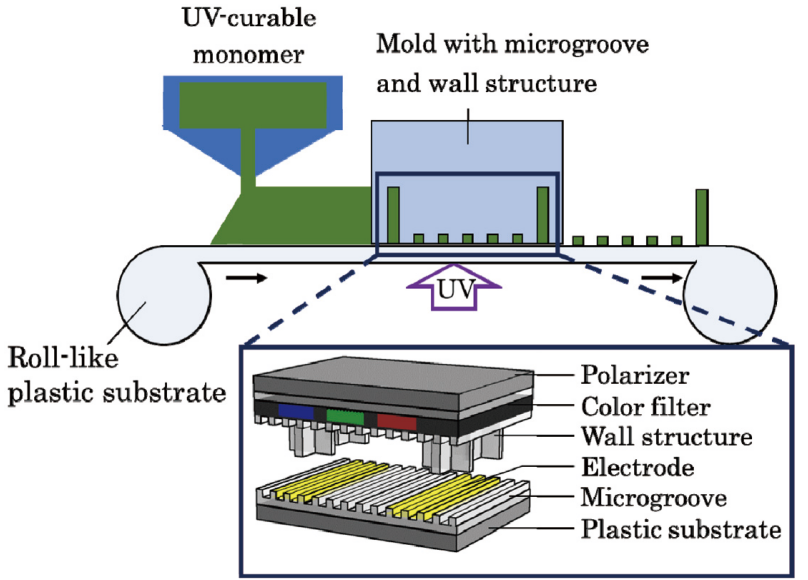

Fig.1 Proposed manufacturing method of IPS-mode LCDs.

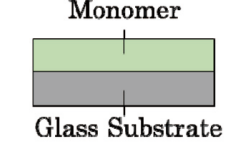

(a) Monomer coating

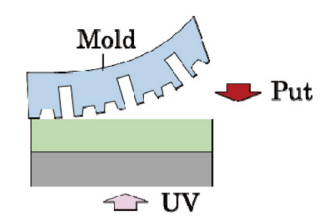

(b)Putting mold and UV light exposure

Fig.2 Fabrication process of microgroove and wall structure.

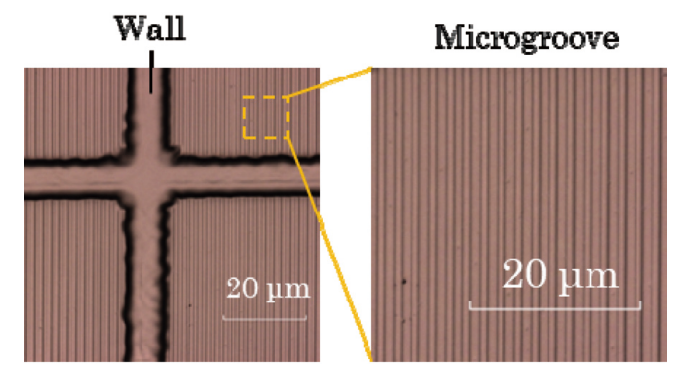

Fig.3 Confocal laser microscope images of transferred polymer surface.

Table 1 Estimated dimensions of microgroove and wall structure from confocal laser microscope images.

\begin{tabular}{lrrrr}
\hline & \multicolumn{2}{c}{ Wall } & \multicolumn{2}{c}{ Microgroove } \\
\hline & Mold & Polymor & Mold & \multicolumn{1}{c}{ Polymer } \\
\hline Depth & 2.49 & 2.42 & 0.18 & 0.18 \\
Width & 9.65 & 9.56 & 1.96 & 1.96 \\
\hline & & & & \\
{$[\mu \mathrm{m}]$}
\end{tabular}

confirmed, and the estimated dimensions are summarized in Table 1; these are consistent with those of the silicone elastomer mold. It was hence confirmed that the fabrication of accurately transferred microgroove and wall structure without air bubbles or contamination in the interface between the mold and monomer.

\section{Voltage-transmittance characteristics}

Fig. 4 shows the fabrication procedure for IPS-mode 


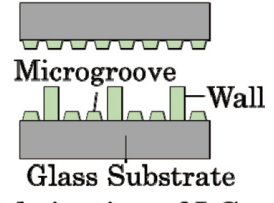

(a) Fabrication of LC cells

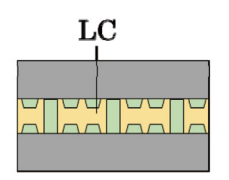

(b) LC injection

Fig.4 Fabrication process of LC devices with microgroove and wall structure.

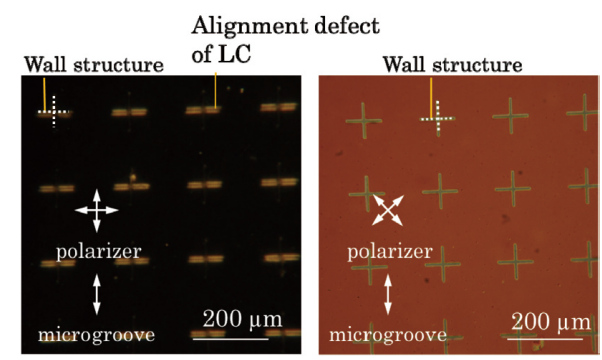

Fig.5 Crossed-Nicols polarizing microscope images of LC device with microgroove and wall structure.

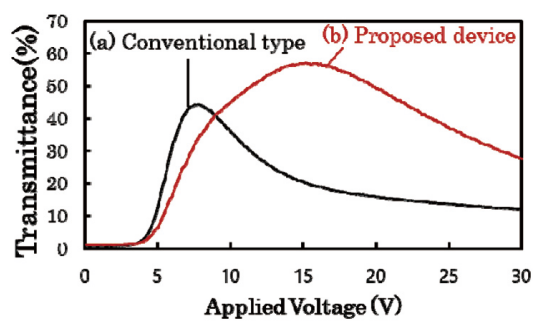

Fig.6 Voltage-transmittance characteristics of IPS mode LC devices.

LC devices with a microgroove and wall structure. We constructed the cell-type structure using two substrates with a microgroove and wall structure, as discussed in Section 3; then, we injected nematic-phase LC (TD$5043 \mathrm{XX}$; JNC) into the cell, utilizing capillary action. Figure 5 shows crossed-Nicols polarizing microscope images of the fabricated LC cells. Although light leakage due to the alignment defect of the LC at the wall structure interface was observed, other regions exhibited a uniform dark state. When the microgroove direction and polarization direction are inconsistent, bright state was observed. It was hence confirmed that the LC molecules aligned along the microgroove direction.

To investigate the electro-optical characteristics of this cell structure, the voltage-transmittance curves of the structurally transferred LC devices were measured, as shown in Fig. 6; the characteristics of conventional IPS-mode LC devices with polyimide alignment layer and spacer structure of $4 \mu \mathrm{m}$ diameter glass beads is also shown for comparison. The driving voltage of the proposed LC device was higher than that of the conventional LC device. To discuss the mechanism of

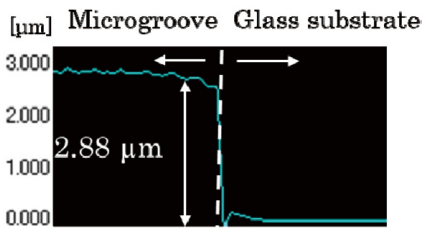

Fig.7 Cross-sectional image of the interface between the microgroove and the glass substrate.

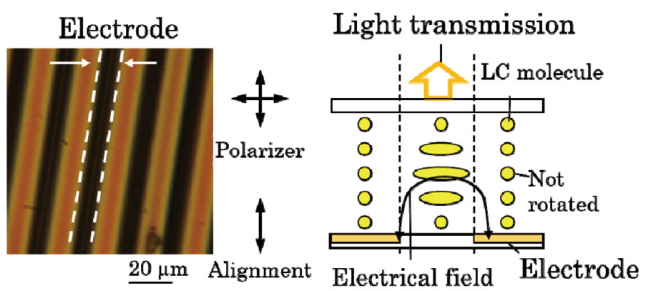

(a) Conventional type

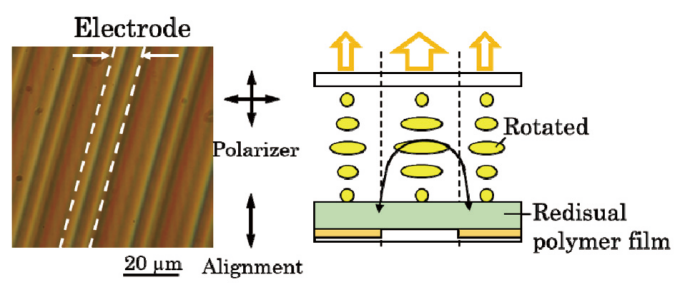

(b) Proposed device

Fig.8 Microscope images and schematic of in-plane switching (IPS)-mode LC devices in the voltage ON state.

high driving voltage, the cross-sectional structure of the proposed LC device was investigated, as shown in Fig. 7. The estimated step height between the glass substrate and microgroove was $2.88 \mu \mathrm{m}$, and this can be attributed to the residual polymer layer on the substrate. The effective electrical field to the LC molecules is reduced by the presence of this residual polymer film; therefore, it is necessary to reduce the thickness of the residual polymer layer to decrease the driving voltage.

In contrast, the maximum transmittance of the proposed LC devices was higher than that of the conventional type. High transmittance in LCDs improves light utilization efficiency. However, minimum transmittance was also higher because of alignment defect. The calculated contrast ratio of conventional LC device was 57.1, which was 51.3 in proposed LC device. Figure 8 shows crossed-Nicols polarizing microscope images of the IPS-mode LC devices in the voltage ON state. Compared to the conventional device, the combshaped electrode region in the proposed device exhibits a bright state. This is caused by rotation of the LC molecules on the electrode due to the weak anchoring energy of the microgrooves ${ }^{12)}$, and/or to extension of the electrical field distribution due to the residual polymer film. To investigate the effect of these factors on the 


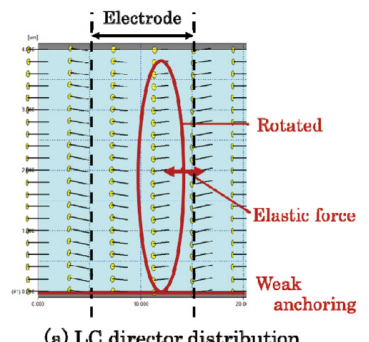

(a) LC director distribution



(b) Transmittance distribution
Fig.9 Simulation result of LC direction and transmittance distribution under weak anchoring.

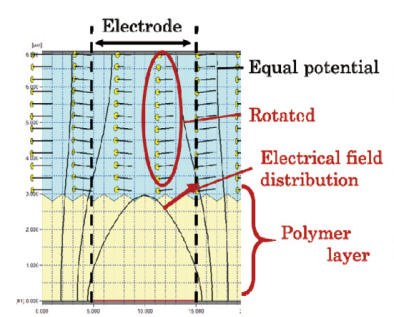

(a) LC director distribution

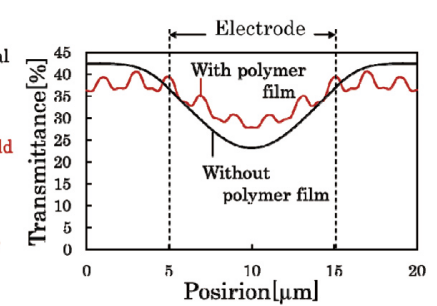

(b) Transmittance distribution
Fig.10 Simulation result of LC direction and transmittance distribution with polymer film.

Table 2 Simulation parameters for TD-5043XX.

\begin{tabular}{|c|c|}
\hline Elastic constant of splay $\left(\mathrm{K}_{11}\right)$ & $8.42 \mathrm{pN}$ \\
\hline Elastic constant of twist $\left(\mathrm{K}_{22}\right)$ & $2.80 \mathrm{pN}$ \\
\hline Elastic constant of bend $\left(\mathrm{K}_{33}\right)$ & $11.66 \mathrm{pN}$ \\
\hline Value of dielectric anisotropy $(\Delta \varepsilon)$ & 3.42 \\
\hline Value of refractive index anisotropy $(\Delta \mathrm{n})$ & 0.135 \\
\hline
\end{tabular}

transmittance individually, we simulated the transmittance and LC alignment distribution based on elastic continuum theory, as shown in Figs. 9 and 10. Figure 9 shows the dependence of the transmittance on anchoring energy. In this simulation, strong and weak anchoring energies were set to be $1.0 \times 10^{-3} \mathrm{~J} / \mathrm{m}^{2}$ and 3.1 $\times 10^{-6} \mathrm{~J} / \mathrm{m}^{2}$, respectively. $1.0 \times 10^{-3} \mathrm{~J} / \mathrm{m}^{2}$ is anchoring energy of general polyimide alignment layer. The value of the weak anchoring energy was adopted from calculated values based on Berreman's theory ${ }^{13)}$. Other physical parameters for the simulation are summarized in Table 2. Figure 9 (a) shows the direction distribution of the LC molecules on a comb-shaped electrode in the voltage ON state. The rotation behavior of LC molecules on electrodes can be observed through the elastic force arising from rotated LC molecules between electrodes. Compared to a strong anchoring energy, the transmittance increased under a weak anchoring energy, as shown in Fig. 9 (b). Here we evaluated response time for turn-on and turn-off processes of each devices, as shown in Table 3. Weak anchoring energy of microgroove caused longer turn-off time than that of
Table 3 Response time of conventional LC device and the proposed LC device.

\begin{tabular}{lrr}
\hline & $\begin{array}{r}\text { Conventional } \\
\text { device }\end{array}$ & $\begin{array}{r}\text { Proposed } \\
\text { device }\end{array}$ \\
\hline Turn-on & 13.2 & 11.0 \\
Turn-off & 12.4 & 229 \\
\hline & & {$[\mathrm{ms}]$}
\end{tabular}

proposed LC device. Figure 10 shows the effect of the residual polymer film on the direction distribution and transmittance. In this simulation, we defined microgroove with width of $2 \mu \mathrm{m}$ pitch and depth of 0.2 $\mu \mathrm{m}$ on $2.8 \mu \mathrm{m}$ dielectric layer. Here, the anchoring energy was assumed to $3.1 \times 10^{-6} \mathrm{~J} / \mathrm{m}^{2}$, corresponding to weak anchoring effect. From Fig. 10 (a), it was demonstrated that LC molecules were rotated by extension of the electrical field due to the presence of the residual polymer film. As the result transmittance on electrode increased, as shown in Fig 10 (b). From these results, we clarified that the high transmittance of the IPS-mode LC device with the microgroove and wall structure is based on both the weak anchoring energy from the microgroove, and extension of the electrical field by the residual polymer film. Power consumption increases due to increasing of driving voltage, and it decreases due to increasing of maximum transmittance. Since power saving is important for portable and flexible LCDs, it will be necessary to optimize structural parameters for minimization power consumption.

\section{Fabrication of flexible LC device with microgroove and wall structure using plastic substrate}

Figure 11 shows the transfer fabrication procedure for the flexible LC devices with a microgroove and wall structure on a plastic substrate. First, we coated a UV curable monomer material (NOA 63; Norland Ltd.) diluted by toluene (mass ratio $1: 1$ ) on the plastic substrate by spin coating for 150 seconds at 5, $000 \mathrm{rpm}$. Usually, the substrate is fixed at the center of the spin table using vacuum chuck; however, the soft plastic

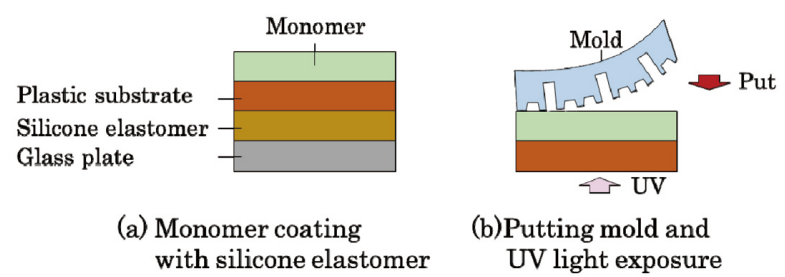

Fig.11 Fabrication process of microgroove and wall structure on plastic substrate. 


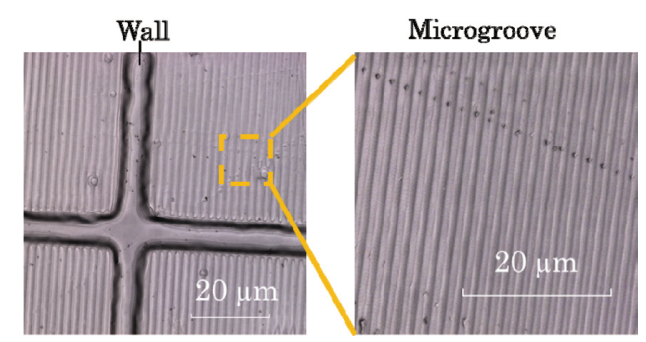

Fig.12 Surface images of fabricated polymer structure on the plastic substrate.

Table 4 Estimated dimensions of microgroove and wall structure from confocal laser microscope images of the plastic substrate.

\begin{tabular}{lcrrr}
\hline & \multicolumn{2}{c}{ Wall } & \multicolumn{2}{c}{ Microgroove } \\
\hline & Mold & Polymer & Mold & Polymer \\
\hline Depth & 1.54 & 1.52 & 0.20 & 0.21 \\
Width & 8.37 & 8.25 & 1.92 & 1.94 \\
\hline & & & & \\
\hline$\mu \mathrm{m}]$
\end{tabular}

substrate will be deformed by vacuum chuck. To overcome this problem, a glass plate was used to hold the flatness of plastic substrate and silicone elastomer was used as an adhesive buffer plate. Then, the mold with the microgroove and wall structure was placed on the coated monomer, and irradiated with UV light (365 $\mathrm{nm}$ wavelength) for $3 \mathrm{~min}$. Here, UV intensity after passing through plastic substrate was adjusted 100 $\mathrm{mW} / \mathrm{m}^{2}$. Figure 12 shows confocal laser microscope images of the surface of the structurally transferred monomer after UV irradiation. It was confirmed that periodic microgroove and cross-shaped wall structure were formed on the polymer surface. However, structural distortion at microgroove was observed. We consider that this is caused by plastic substrate being soft and distorted. The dimensions of these structures were summarized in Table 4, and there was negligible difference in structural dimensions between the surfaces of the mold and the polymer. We have therefore succeeded in transferring the microgroove and wall structure onto the plastic substrate, as well as onto the glass substrate, as described in Section 3.

Figure 13 shows the fabrication procedure for the LC devices with the microgroove and wall structure using a plastic substrate. The LC cell structure was constructed using two structurally transferred plastic substrates, as shown in Fig.13 (a); then, we injected LCs (TD-5043XX, JNC) into the cells, utilizing capillarity action. CrossedNicols polarized microscope images of the fabricated LC device are shown in Fig. 14. When the direction along the microgrooves is matched to the transmission axis of

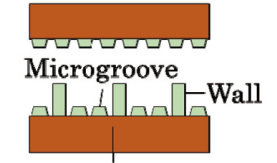

Plastic substrate

(a) Fabrication of LC cells using plastic substrate

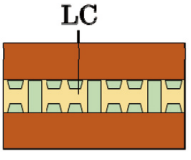

(b) LC injection
Fig.13 Fabrication process of LC device with plastic substrate.

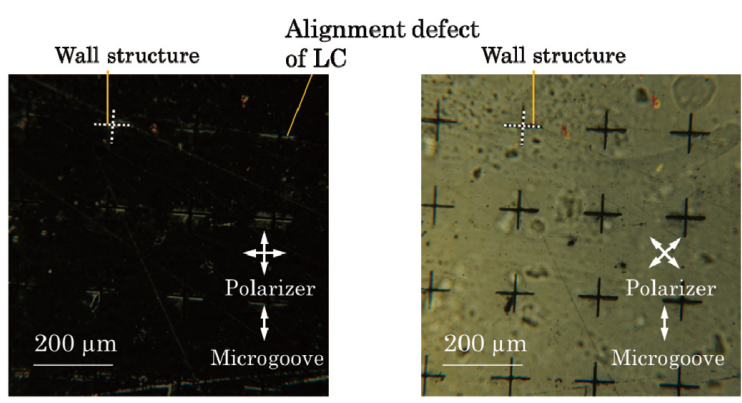

Fig.14 Crossed-Nicols polarizing microscope images of LC device with microgroove and wall structure using plastic substrate. The described cross-like white dotted line represents a wall structure.

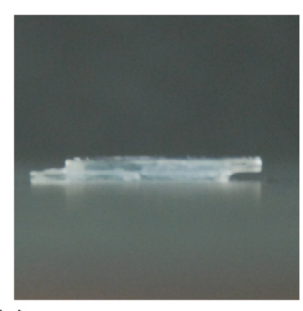

(a) LC device using glass substrate

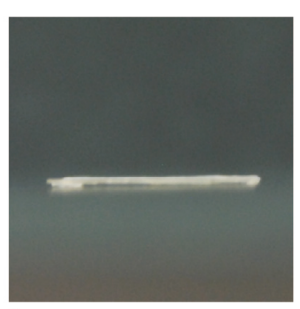

(b) LC device using plastic substrate
Fig.15 Side images of LC device using glass substrate and plastic substrate.

the polarizer, light leakage caused by the alignment defect of LCs was observed at the wall structure interface. Most regions on the microscope image exhibited a uniform dark state. With a 45 degree rotation of the crossed-Nicols polarizer, a bright state was obtained in all regions, except for at the wall structures. However, alignment defect was observed in bright region. Distortion of microgroove is considered as this cause. These results indicate that LC molecules were aligned parallel to the microgrooves. In addition, the flexible LC device using plastic substrate was thinner than LC device using glass substrate, as shown in Fig. 15. In this study, we have demonstrated that the transfer method for the microgroove and wall structure can also be applied to a flexible plastic substrate. Hence, we showed the possibility of realization of largearea flexible LCDs with improved portability. 


\section{Conclusions}

In this study, we proposed a fabrication method using transferred formation of microgroove and wall structure for flexible IPS-mode LC devices. For demonstration of the proposed method, we fabricated an IPS-mode LC device with a microgroove and wall structure, which exhibited higher transmittance and driving voltage than conventional IPS-mode LC devices. We clarified that a reduction in the thickness of the residual polymer film was necessary to decrease the driving voltage based on LC elastic continuum theory. In addition, we succeeded in fabricating flexible LC devices with a microgroove and wall structure on a plastic substrate, and confirmed that the transfer technique for the microgroove and wall structure can be applied for fabrication of flexible LC devices using a plastic substrate.

\section{References}

1) Sato, H. et al. A4-sized Liquid Crystal Displays with Flexible Light Guide Plate. IDW'06 605-608 (2006)

2) Fujikake, H. et al. Flexible Field-Sequential-Color FLC Displays Using a Bendable Backlight Sheet with LED Chips. IDW/AD'05 871-874 (2005)

3) Lee, W., Lee, W. \& Lu, Y. Fast-Switching Stratified PolymerStabilized Liquid Crystal for Flexible Display Applications. Polymer (Guildf). 348-350

4) Ahn, S. H. \& Guo, L.J. Large-area roll-to-roll and roll-to-plate Nanoimprint Lithography: A step toward high-throughput application of continuous nanoimprinting. ACS Nano 3, 2304-2310 (2009)

5) Ahn, S. H. \& Guo, L.J. High-speed roll-to-roll nanoimprint lithography on flexible plastic substrates. Adv. Mater. 20, 20442049 (2008)

6) Ishinabe, T., Obonai, Y. \& Fujikake, H. A Foldable Ultra-Thin LCD Using a Coat-Debond Polyimide Substrate and Polymer Walls. Proc. Soc. Inf. Disp. 9-1, 83-86 (2016)

7) Obonai, Y., Shibata, Y., Ishinabe, T. \& Fujikake, H. Flexible Liquid Crystal Devices Using Coat-Debond Polyimide Substrates and Adhesive Polymer Spacers. IEICE Trans. Electron. E100-C, 10391042 (2017)

8) Minari, T. et al. Room-temperature printing of organic thin-film transistors with ÉE-junction gold nanoparticles. Adv. Funct. Mater. 24, 4886-4892 (2014)

9) Kim, Y. T., Hwang, S., Hong, J. H. \& Lee, S.D. Alignment layerless flexible liquid crystal display fabricated by an imprinting technique at ambient temperature. Appl. Phys. Lett. 89, 88-91 (2006)

10) Chiou, D. R., Chen, L. J. \& Lee, C.D. Pretilt angle of liquid crystals and liquid-crystal alignment on microgrooved polyimide surfaces fabricated by soft embossing method. Langmuir 22, 9403-9408 (2006)

11) Park, M., Son, K., Kim, M. \& Kim, H. 2D-3D Switchable AutoStereoscopic Multi-View 3D Mobile Display Using Polarization Dependent Reactive Mesogen Lens Array Film. 177-178 (2015)

12) Maeda, T. et al. Electro-optic Characteristics of OZ-IPS LCD Utilizing an Application-Type, Zero-Azimuth Anchoring Material. SID Symp. Dig. Tech. Pap. 48, 704-707 (2017)

13) Berreman, D. Solid Surface Shape and the Alignment of an Adjacent Nematic Liquid Crystal. Phys. Rev. Lett. 28, 1683-1686 (1972)

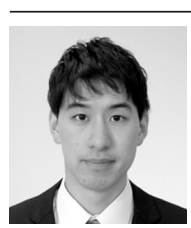

Daisuke Minami received a B.E. degree from Tohoku University, Sendai, Japan, in 2017. He is now a graduate student in the Department of Electronic Engineering at the Graduate School of Engineering, Tohoku University, where he works on the development of flexible LCDs. He received the Encouragement Prize of Student Awards from IEEE Sendai Section in 2017

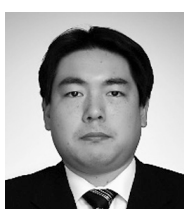

Yosei Shibata received his $\mathrm{PhD}$ degree in engineering from Tokyo Institute of Technology (Japan) in March 2013. He has research interests on organic semiconductor devices such as organic transistors and organic photovoltaics. Then he joined the National Institute of Advanced Industrial Science and Technologies (AIST, Japan) as a postdoctoral position. In October 2015, he joined the Department of Electronics of Tohoku University as an Assistant Professor.

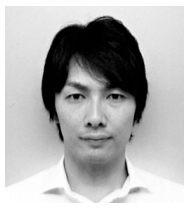

Takahiro Ishinabe received his B.S., M.S. and $\mathrm{Ph}$. D. degrees in Electronic Engineering from Tohoku University, Sendai, Japan, in 1995, 1997 and 2000 , respectively. From 2000 to 2002 , he was a Research Fellow of the Japan Society for the Promotion of Science and from 2003 to 2012, he was an Assistant Professor, and since 2013, he has been an Associate Professor in the Department of Electronics, Graduate school of Engineering, Tohoku University. He has also been a Visiting Professor in the CREOL, The College of Optics and Photonics, University of Central Florida from 2010 to 2011. He has been performing a research on advanced liquid crystal displays such as wide viewing angle $L C D$, reflective full-color LCD, field sequential color LCD and flexible LCD.

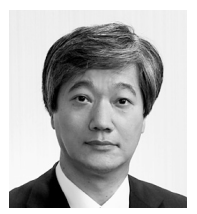

Hideo Fujikake received M.E and Ph.D degrees from Tohoku University, Japan, in 1985 and 2003 , respectively. In 1985 , he joined Japan Broadcasting Corporation (NHK). In 1988-2012, he was with NHK Science and Technology Research Laboratories. Since 2012, he has been a professor at Department of Electronic Engineering, Tohoku University. He received Best Paper Award from Institute of Electronics, Information and Communication Engineers (IEICE) in 2001, Best Paper Award from Japanese Liquid Crystal Society (JLCS) in 2001 and 2015, Niwa-Takayanagi Best Paper Awards from Institute of Image Information and Television Engineers of Japan (ITE) in 2003 and 2009, and Electronics Society Award from IEICE in 2013. His current interest is concerned with flexible liquid crystal displays. He also served as a General Vice Chair in International Display Workshops in 2015-2016, a Japan Chapter Chair in IEEE Consumer Electronics Society in 2012-2014, and a Vice President of Japanese Liquid Crystal Society in 2015-2016. He is an IEICE fellow since 2015, and ITE fellow since 2016. 